\title{
Phenotypic Transition of the Collecting Duct Epithelium in Congenital Urinary Tract Obstruction
}

\author{
Peter Trnka, ${ }^{1}$ Michael J. Hiatt, ${ }^{1}$ Larissa Ivanova, ${ }^{1}$ Alice F. Tarantal, ${ }^{2,3}$ \\ and Douglas G. Matsell ${ }^{1,2,4}$ \\ ${ }^{1}$ Child and Family Research Institute, University of British Columbia, Vancouver, BC, Canada V5Z 4 H4 \\ ${ }^{2}$ Center of Excellence in Translational Human Stem Cell Research, University of California, Davis, California, CA 95817, USA \\ ${ }^{3}$ California National Primate Research Center, Departments of Pediatrics and Cell Biology and Human Anatomy, \\ University of California, Davis, California, CA 95616, USA \\ ${ }^{4}$ Division of Nephrology, BC Children's Hospital, 4480 Oak Street, Vancouver, BC, Canada V6H 3 V4
}

Correspondence should be addressed to Douglas G. Matsell, dmatsell@cw.bc.ca

Received 26 June 2009; Revised 10 September 2009; Accepted 11 September 2009

Academic Editor: Kenichiro Kitamura

Copyright (c) 2010 Peter Trnka et al. This is an open access article distributed under the Creative Commons Attribution License, which permits unrestricted use, distribution, and reproduction in any medium, provided the original work is properly cited.

Epithelial-mesenchymal transition (EMT) has emerged in recent years as an important process in the development of organ fibrosis in many human diseases. Our previous experience in a nonhuman primate model of obstructive nephropathy suggested that EMT of collecting duct epithelium contributes to the development of interstitial fibrosis. In this study we demonstrate for the first time in humans that obstructed fetal collecting duct epithelium undergoes transition to mesenchymal phenotype, characterized by decreased expression of epithelial markers, de novo expression of mesenchymal markers with subsequent loss of cell-cell interaction, disruption of the basement membrane, and increased deposition of extracellular matrix into the expanded interstitium of the obstructed kidney. The results of this study therefore support the previous findings from animal studies and suggest that EMT of the collecting duct epithelium might contribute to the development of interstitial fibrosis in human fetal obstructive nephropathy.

\section{Introduction}

Renal hypoplasia/dysplasia, often associated with obstructive nephropathy, is the leading cause of end stage renal disease in childhood [1]. Despite advances in diagnosis and antenatal management of congenital urinary tract obstruction, the outcomes of these children have not improved since fetal intervention was introduced into clinical practice over 20 years ago.

Injury to the epithelium in a number of human disease states causes epithelial cells to acquire mesenchymal characteristics through the process of epithelial-mesenchymal transition (EMT), and in congenital urinary tract obstruction this contributes to the characteristic tubulointerstitial disease [2-7]. The hallmark of renal dysplasia is the accumulation of extracellular matrix in the interstitium leading to fibrosis. The injured epithelium plays an important role in this process.
We have previously studied the pathogenesis of renal dysplasia in a nonhuman primate model of obstructive nephropathy $[8,9]$, and highlighted significant histopathological changes in the renal medullary interstitium, including dilatation of the collecting ducts (CDs), and disruption of the $\mathrm{CD}$ epithelium. We have also defined features suggestive of CD EMT in this experimental animal model with loss of the normal epithelial phenotype, gain of a mesenchymal phenotype, disruption of the tubular epithelial basement membrane, and migration of the transformed cells into the interstitium [10].

The aim of this study was to test the hypothesis that congenital urinary tract obstruction in humans results in CD epithelial cell injury and transition to a mesenchymal phenotype, and that these transformed cells contribute to interstitial fibrosis. 


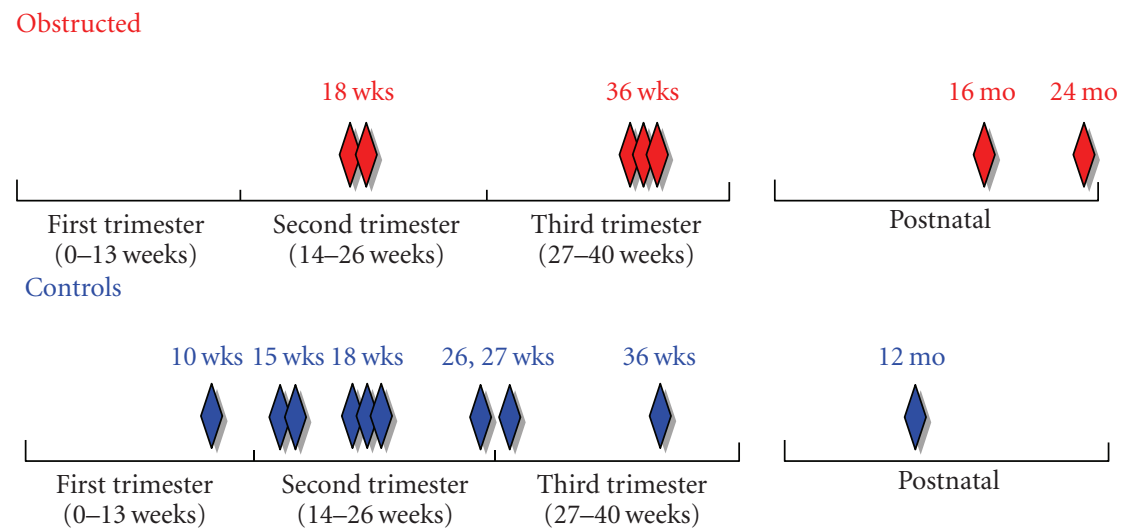

FIGURE 1: Gestational and postnatal ages of the obstructed and control kidney tissue samples.

\section{Materials and Methods}

2.1. Tissue Samples. Specimens from obstructed and normal human fetal and postnatal kidneys were studied (total $n=$ 17). Obstructed kidney samples were obtained from fetuses in the second (18 weeks gestation, $n=2$ ) and third trimesters (36 weeks gestation, $n=3$ ), and postnatal ages (16 and 24 months, $n=2$ ). Controls consisted of kidney samples from fetuses in the late first trimester (10 weeks, $n=1)$, second trimester ( 15,18 and 26 weeks, $n=6)$, and third trimester (27 and 36 weeks gestation, $n=2$ ), and 12 months postnatal age $(n=1)$ (Figure 1). In all cases fetuses had no other noted anomalies. Control kidney samples were obtained from aborted fetuses and children that died from causes unrelated to renal disease. All samples were collected in accordance with the ethical guidelines of the University of British Columbia, and all performed analyses were approved by the Ethics Committee of the University of British Columbia.

2.2. Tissue Processing. After collection, kidneys were sectioned transversely and sagitally then immediately fixed in 4\% paraformaldehyde overnight, and dehydrated through graded ethanol before transfer to toluene. Sections of paraffin-embedded tissue were cut with a microtome (5$10 \mu \mathrm{m}$ ), mounted on Superfrost slides (VWR), and baked overnight at $55^{\circ} \mathrm{C}$. Sections were then cooled to room temperature and stored with desiccant. Samples for realtime PCR were snap frozen in liquid nitrogen and stored at $\leq-80^{\circ} \mathrm{C}$.

2.3. Immunofluorescent Histochemistry. Tissue sections were deparaffinized in xylene and rehydrated by passage through graded ethanol. For light microscopy, sections were stained with hematoxylin for 2 minutes and eosin for 40 seconds. For immunohistochemistry, slides were subjected to 40 minutes of heat-induced epitope retrieval in $10 \mathrm{mM}$ citrate buffer ( $\mathrm{pH}$ 6-6.5). Sections were subsequently cooled to room temperature and blocked for 1 hour with $2 \%$ goat or donkey serum. Following blocking, excess buffer was removed from the sections and replaced with diluted primary antibodies in staining buffer for incubation overnight at $4^{\circ} \mathrm{C}$. Sections were then incubated for 1 hour with fluorescently conjugated secondary antibodies at room temperature. Nuclei were also stained for 5 minutes with DAPI dilactate ( $1: 36$, Invitrogen) prior to mounting with Prolong Gold mounting media with antifade (Invitrogen). Analysis of tissue staining was performed on a Leica epifluorescence microscope (Wetzlar, Germany) using images captured with a Retiga 1300i camera (QImaging, Canada) and processed with OpenLab imaging software (Improvision, MA, USA). Primary antibodies and their dilutions were as follows: anticytokeratin (DakoCytomation, Carpinteria, CA), antiE-cadherin (BD Transduction Laboratories, San Diego, CA), anti $\beta$-catenin (Cell Signalling Technology Danvers, MA), anti $\alpha$-smooth muscle actin, anticollagen IV (Fitzgerald Industries International Inc., Concord, MA), and antivimentin (Sigma-Aldrich, Saint Louis, MO) (all diluted 1 : 100). Secondary antibodies included goat antimouse Alexa488, goat antirabbit Alexa568, and donkey antigoat Alexa568 (Invitrogen Canada Inc., Canada) (all diluted 1 : 400). For correlation between the degree of obstruction, and periductal $\alpha$-SMA and CD epithelial vimentin expression, we measured the areas of the CD dilatation, the areas of $\alpha$-SMA in periductal collars, and the areas of the CD cells expressing vimentin (all expressed in $\left.\mathrm{mm}^{2}\right)$ in obstructed fetal and postnatal kidneys $(n=3)$ using the OpenLab imaging software. The relationship between measured areas was expressed as a scatter plot with trend lines using Microsoft Excel 2008 for Mac.

2.4. Real-Time PCR. Real-time PCR analysis was performed on total RNA extracted from normal kidneys at 10 weeks gestation $(n=1), 15$ weeks gestation $(n=2)$, and 18 weeks gestation $(n=1)$, and obstructed kidneys at 18 weeks gestation $(n=2)$ and 36 weeks gestation $(n=1)$. Total RNA was isolated from the whole kidneys using RNeasy Mini Kit protocol (Qiagen Inc., Mississauga, ON, Canada) with RNA-free DNase treatment before treatment with PowerScript reverse transcriptase (BD Biosciences, San Jose, CA). Predesigned TaqMan Gene Expression Assays were used for the following target genes: E-cadherin (Hs01013953_m1), Ncadherin (Hs00983062_m1), $\alpha$-catenin (Hs00426996_m1), 


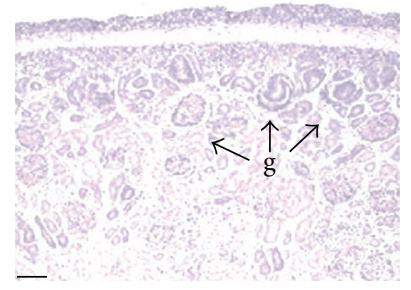

(a)

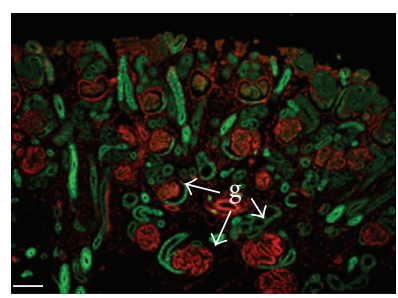

(e)

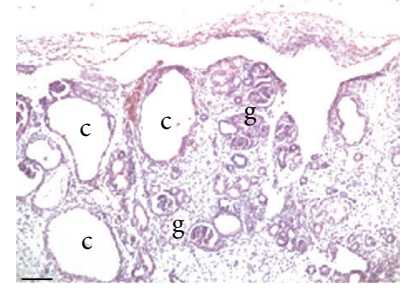

(b)

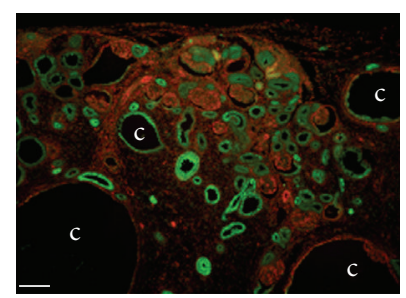

(f)

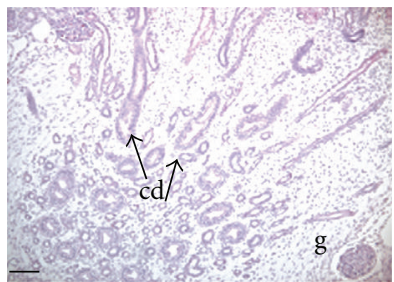

(c)

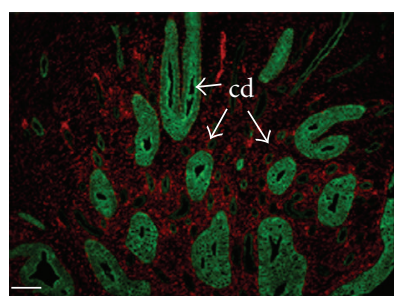

(g)

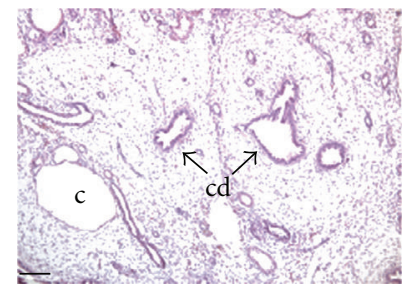

(d)

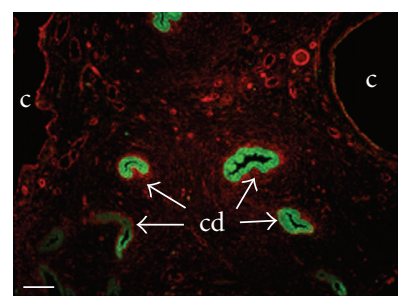

(h)

FIGURE 2: Histological features of human fetal obstructive nephropathy. Obstructed 18 weeks gestation kidney shows disorganized cortical architecture with multiple glomerular cysts (c), decreased glomerular (g) endowment ((b) and (f)), and reduced number and dilation of the collecting ducts (cd) in the hypoplastic medulla ((d) and (h)). Cortex ((a) and (e)) and medulla ((c) and (g)) of a normal 18-week gestation kidney. (a)-(d): hematoxylin and eosin stain; (e)-(h) immunofluorescence histochemistry: green $=$ cytokeratin, red $=$ vimentin. Scale bar $=$ $100 \mu \mathrm{m}$.

$\beta$-catenin (Hs00355045_m1), vimentin (Hs00958116_m1), alpha-3 chain of type IV collagen (Hs01022542_m1), alpha-4 chain of type IV collagen (Hs01011885_m1), $\alpha$ smooth muscle actin (Hs00559403_m1), and cyclophilin A (Hs99999904_m1) (Applied Biosystems, Foster City, CA). All samples were amplified in triplicate on ABI Prism 7000 Sequence Detection System using TaqMan Universal PCR MasterMix (Applied Biosystems, Foster City, CA). Optimization and relative quantification were done using the prevalidated comparative CT method. Normalization of RNA quantity between samples was accounted for using the expression of the housekeeping gene cyclophilin A. The fold change of each gene between samples was determined relative to control kidney at 18 weeks gestation.

\section{Results}

3.1. Histological Features of Human Congenital Obstructive Dysplasia. Congenital urinary tract obstruction was associated with abnormal ureteric bud branching and defective metanephric mesenchyme induction with subsequent abnormal collecting duct, tubular, and glomerular development (Figure 2). All obstructed developing kidneys showed focal areas of dysplastic and cystic changes. Multiple glomerular cysts were identified in both the cortex and the medulla, whereas cystic dilatation of the renal tubules and CDs was seen predominantly in the medulla (Figures 2(b) and $2(\mathrm{~d})$ ). The most pronounced dysplastic changes were seen in the medullary regions of the obstructed kidneys where some areas showed complete loss of normal structure with dilated CDs surrounded by thick mesenchymal collars. Other histopathological features of dysplasia included decreased glomerular endowment, reduced number of CDs, and medullary hypoplasia with interstitial expansion and interstitial fibrosis. The most dilated CDs were lined with a flattened epithelium (Figure 2). These changes were present from early gestation and persisted postnatally.

\subsection{Loss of Epithelial Phenotype in the Obstructed Collecting} Duct. The expression of the cytoskeletal proteins E-cadherin and $\beta$-catenin was studied in normal and obstructed human fetal kidneys. These proteins are important in normal development of the kidney epithelium [11] and are responsible for the maintenance of cell polarity, cell-cell adhesion, and epithelial integrity [12]. In the normal human $\mathrm{CD}$ epithelium, E-cadherin and $\beta$-catenin were expressed early in gestation. Developmentally younger kidneys had more pronounced cytoplasmic expression of both proteins (Figure 3(a), inset), whereas kidneys from mid to late gestation and postnatal kidneys showed colocalization of E-cadherin and $\beta$-catenin at the intercellular junctions of adjacent CD epithelial cells (Figures 3(b) and 3(c)). In the obstructed kidneys, the disruption of E-cadherin- $\beta$ catenin colocalization was noted, as well as a loss of Ecadherin and $\beta$-catenin from the cellular membranes, and the translocation of these proteins into the cytoplasm, with E-cadherin identified in the nucleus of the injured CD epithelial cells (Figures 3(d), 3(e), and 3(f)).

\subsection{Gain of Mesenchymal Phenotype in the Obstructed} Collecting Duct. Injured epithelial cells undergoing EMT express mesenchymal markers, including fibroblast-specific protein-1, $\alpha$-smooth muscle actin ( $\alpha$-SMA), and vimentin [13]. We have previously demonstrated de novo $\alpha$-SMA expression in CD epithelial cells in the obstructed nonhuman primate [10]. These cells contribute to the development 


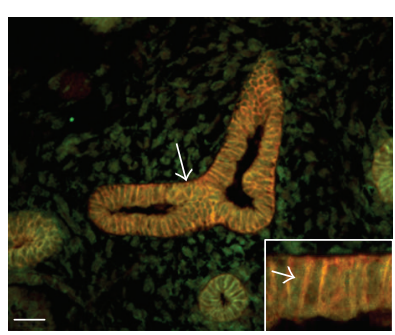

(a)

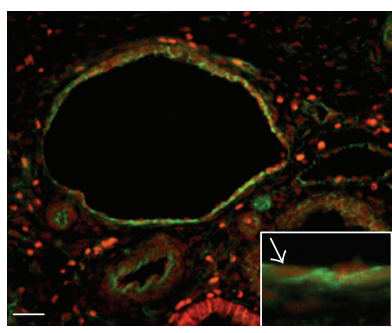

(d)

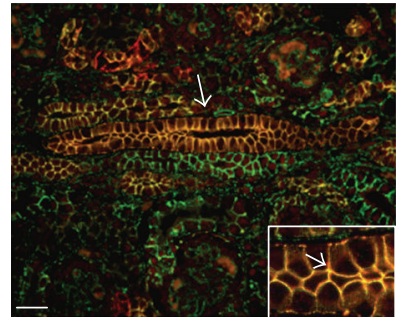

(b)

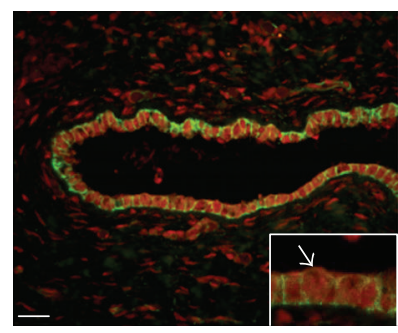

(e)

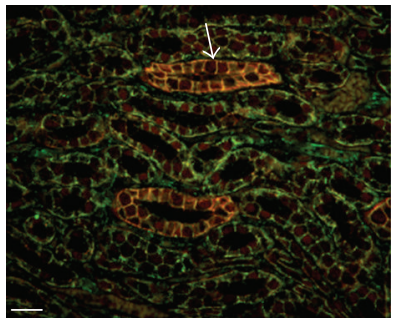

(c)

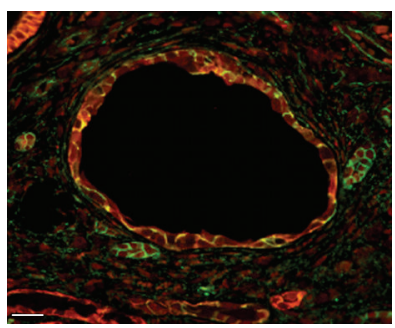

(f)

FIGURE 3: E-cadherin and $\beta$-catenin immunoreactivity (IR). Normal kidneys ((a)-(c)). (a) In the early gestation kidney (10 weeks), E-cadherinIR (red) and $\beta$-catenin-IR (green) are localized in the cytoplasm (arrows). (b) Colocalization of E-cadherin-IR and $\beta$-catenin-IR (yellow) to the intercellular junctions is well established by late gestation (27 weeks) (arrows), and (c) persists in the normal postnatal (12 months) kidney (arrow). Obstructed kidneys ((d)-(f)). (d) Disruption of E-cadherin-IR and $\beta$-catenin-IR colocalization at the intercellular junctions, with translocation of $\beta$-catenin-IR to the cytoplasm (green) and of E-cadherin-IR to the nucleus (red) (arrows in inset) in the (d) mid gestation kidney (18 weeks), (e) late gestation kidney (36 weeks), and (f) postnatally (16 months kidney). Scale bar $=25 \mu \mathrm{m}$.

of immature mesenchymal collars surrounding the dilated ducts. In the human fetal kidneys examined in this study, the normal CD epithelium did not express $\alpha$-SMA at any stage of gestation or postnatally (Figures $4(\mathrm{a}), 4(\mathrm{~b})$, and $4(\mathrm{c}))$. However, we observed de novo $\alpha$-SMA expression in the obstructed epithelium at mid gestation, but not later in gestation or postnatally (Figure 4(d)). Mid gestation obstructed kidneys developed peritubular circumferential cellular collars expressing $\alpha$-SMA (Figure $4(\mathrm{~d})$ ), becoming more pronounced as gestation progressed (Figure 4(e)). The most extensive collars were observed in the inner medulla of postnatal kidneys (Figure 4(f)) and also in the most severely obstructed fetal kidneys. The severity of periductular tubular collar formation correlated with the extent of epithelial injury and the severity of the obstruction.

Vimentin, a member of the intermediate filament family of proteins, offers flexibility to the cell while maintaining cell shape and cytoskeletal integrity [14]. Under normal circumstances, differentiated epithelial cells do not express appreciable amounts of vimentin, whereas expression of this protein is a characteristic feature of myofibroblasts. In normal fetal kidneys, interstitial cells expressed vimentin, which was most pronounced in younger gestation kidneys, decreasing in later gestation, and almost absent in postnatal kidneys (Figures 5(a), 5(b), and 5(c)). As expected, normal CD epithelial cells did not express vimentin. However, obstructed CD epithelial cells showed a very pronounced de novo expression of vimentin that correlated with the severity and the duration of the obstruction, increasing from mid to late gestation and postnatally (Figures 5(d), 5(e), and 5(f)). Additionally, obstructed fetal kidneys developed an expanded interstitium, which expressed abundant vimentin, compared to normal kidneys of similar gestation. This was more pronounced in younger kidneys.

\subsection{Disruption of the Basement Membrane of the Obstructed} Collecting Duct. Type IV collagen (ColIV) is a major structural component of all basement membranes, including the CD basement membrane. ColIV molecules are synthesized by the cells attached to the basement membrane and are subsequently incorporated into the membrane itself [15]. In EMT, the disruption of basement membranes is necessary to allow the migration of the transformed tubular epithelial cells [4]. In this study, the CD epithelium of normal kidneys expressed cytoplasmic ColIV diffusely at mid gestation and apically at late gestation (Figures 5(a) and 5(b)). There was no epithelial expression of ColIV in normal postnatal kidneys (Figure 5(c)). Notably, and as expected, all normal fetal and postnatal kidneys expressed ColIV in the CD basement membranes in a thin, regular, and well-defined pattern (Figures 5(a), 5(b), and 5(c)). In addition to an increase in apical epithelial cell expression of ColIV, obstructed kidneys demonstrated a marked disruption of the CD basement membrane, characterized by thickening, attenuation, and loss of ColIV expression (Figures 5(d), 5(e), 5(f)). Interestingly, the expanded interstitium of the obstructed fetal and postnatal kidneys expressed an abundance of ColIV, most pronounced in the immature mesenchymal collars surrounding the most dilated CDs, particularly in the inner medulla of postnatal kidneys, suggesting a correlation with the severity and duration of the obstruction (Figure 5(f)). 


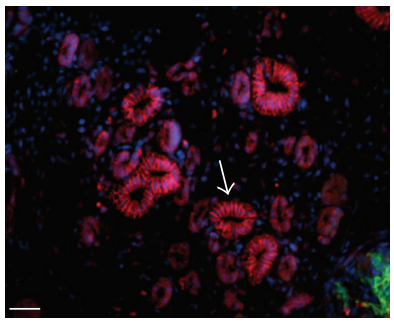

(a)

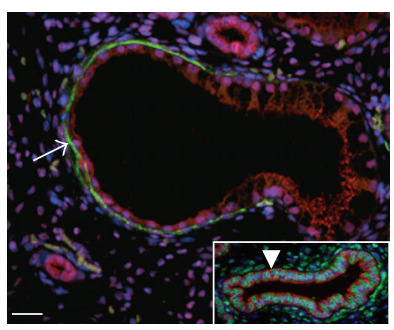

(d)

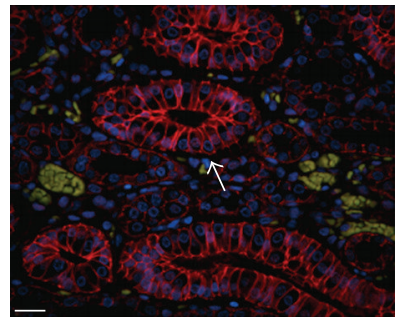

(b)

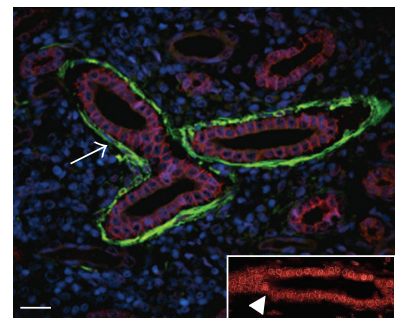

(e)

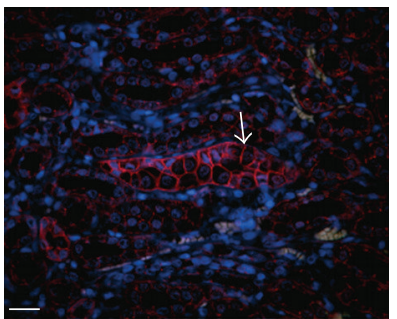

(c)

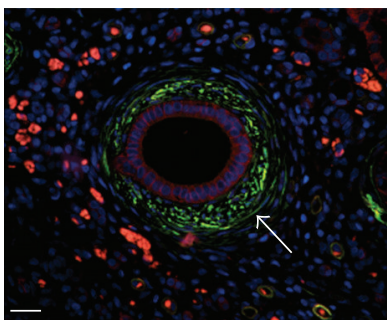

(f)

FIGURE 4: E-cadherin and $\alpha$-smooth muscle actin immunoreactivity. Normal kidneys ((a)-(c)). In the normal mid gestation (18 weeks) (a), late gestation (27 weeks) (b), and postnatal (12 months) (c) kidneys, $\alpha$-smooth muscle actin ( $\alpha$-SMA)-IR (green) was absent in the CD epithelium; however, intercellular localization of E-cadherin-IR (red) was well established from mid gestation onwards (arrows). Obstructed kidneys ((d)-(f)). Circumferential collars expressing $\alpha$-SMA-IR (green) are present in the (d) mid gestation (18 weeks), (e) late gestation (36 weeks), and (f) postnatal (16 months) kidneys (arrows). De novo expression of $\alpha$-SMA-IR in the obstructed CD epithelium is present at 18 weeks gestation ((d) inset, arrowhead) but not in the late gestation (e) or postnatal (f) kidneys. As in Figure 3, E-cadherin expression (red) is disrupted, with translocation from the cell membrane to cytoplasm $((\mathrm{d})-(\mathrm{f}))$ and to the nucleus $((\mathrm{e})$ inset, arrowhead). Scale bar $=25 \mu \mathrm{m}$.

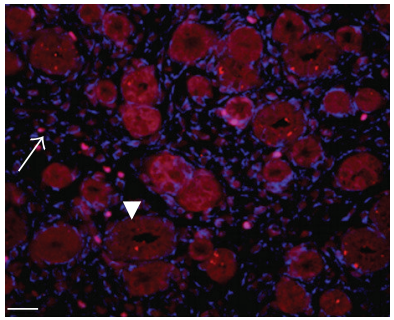

(a)

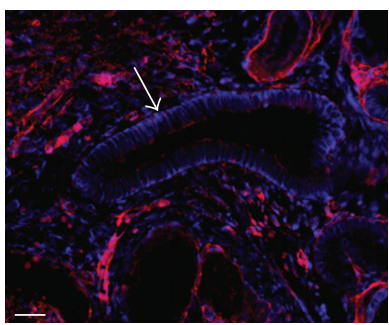

(d)

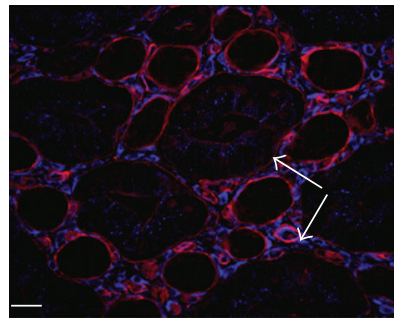

(b)

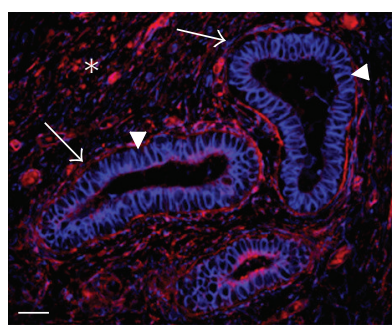

(e)

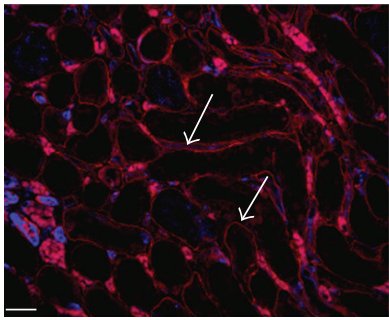

(c)

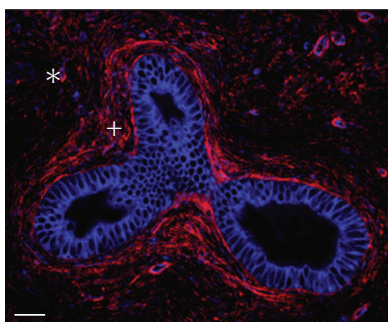

(f)

FIGURE 5: Vimentin and collagen IV immunoreactivity. Normal kidneys ((a)-(c)). (a) Interstitial expression of vimentin-IR (blue) (arrow) and diffuse cytoplasmic expression of collagen IV (ColIV)-IR (red) (arrowhead) are present in the mid gestation (18 weeks) kidney. (b) Thin, regular, well-defined expression of ColIV-IR in the CD basement membranes (arrows) is well established by late gestation (27 weeks), and (c) persists postnatally (12 months) (arrows). Obstructed kidneys ((d)-(f)). (d) De novo basolateral expression of vimentin-IR (blue) (arrow) in the CD epithelium of the obstructed 18 weeks gestation kidney. (e) More pronounced vimentin-IR expression (arrowheads) and disruption of ColIV-IR expression (red) (arrows) in the CD basement membrane of the late gestation (36 weeks) obstructed CD epithelium, and (f) in the obstructed postnatal (16 months) kidneys. Deposition of ColIV-IR in the expanded interstitium (asterisk), and in the periductal collars (plus) is also present. Scale bar $=25 \mu \mathrm{m}$. 


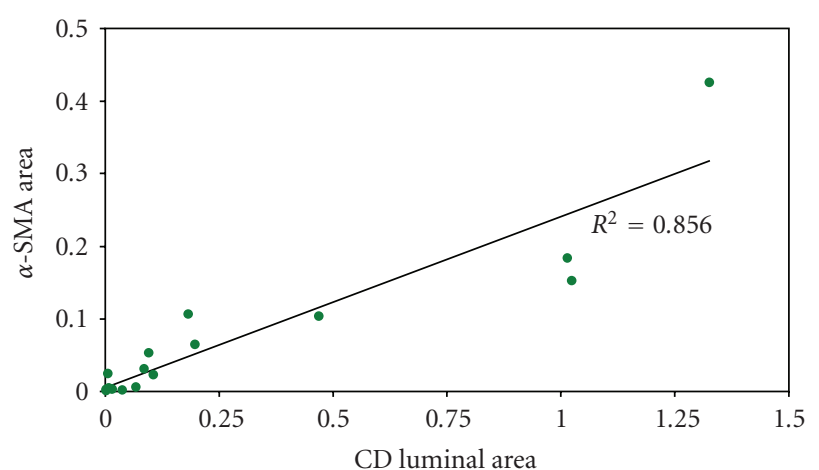

(a)

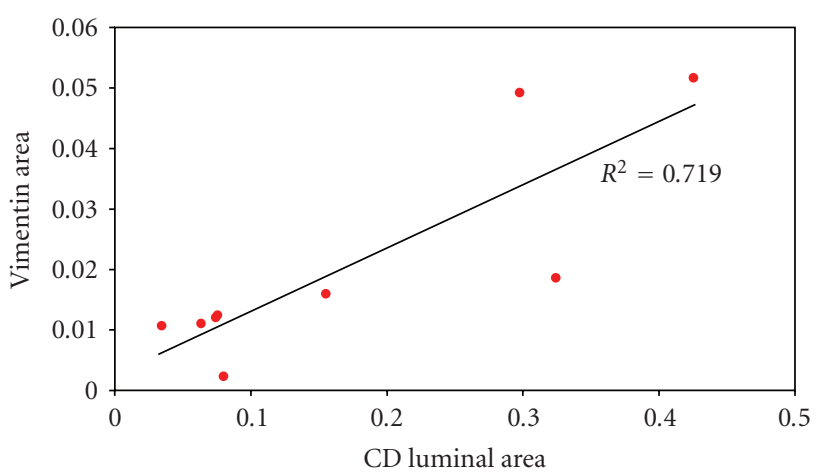

(b)

FIGURE 6: Correlation of the severity of CD dilatation with periductal collar formation and with CD epithelial vimentin expression. The relationship between CD dilatation ( $x$-axis), and the areas of periductal collars expressing $\alpha$-SMA (a) and of CD epithelium expressing vimentin (b) ( $y$-axis) is shown. Measurements were performed in obstructed fetal (36 weeks gestation) and postnatal (16 months) kidneys $(n=3)$. All areas are expressed in $\mathrm{mm}^{2}$.

To show the relationship between the severity of obstruction and the extent of phenotypic transition of the CD epithelium, we correlated CD dilatation (as a marker of severity of obstruction) with the expression of $\alpha$-SMA in periductal collars and of vimentin by the CD cells (as markers of epithelial injury). CD dilatation correlated with periductal $\alpha$-SMA expression and with $\mathrm{CD}$ epithelial cell vimentin expression (Figure 6).

3.5. Gene Expression in Obstructed Fetal Kidneys. Quantitative real-time PCR analysis was performed for genes encoding epithelial and mesenchymal proteins of interest on total RNA samples obtained from normal (early to mid gestation) and obstructed (mid to late gestation) whole kidneys. First, we studied normal changes in gene expression with advancing gestation. Among genes encoding epithelial proteins, E-cadherin mRNA expression increased in the 18 versus 10 weeks gestation kidneys, with little change in $\alpha$ catenin, $\beta$-catenin, and $\mathrm{N}$-cadherin expression (Figure 7(a)). For genes encoding mesenchymal proteins, vimentin, alpha3 and alpha- 4 chains of ColIV, and $\alpha$-SMA were all increased in the normal 18 weeks compared to 10 weeks gestation kidney (Figure 7(a)).

We next studied changes in the mRNA expression of genes encoding epithelial and mesenchymal proteins in mid (18 weeks) and late gestation (36 weeks) obstructed kidneys, using the 18-week gestation normal kidney as the reference control value. Of the genes encoding epithelial proteins, $\mathrm{N}$-cad expression decreased in the late gestation obstructed kidney, while $\alpha$-catenin and $\beta$-catenin mRNA increased (Figure 7(b)). For genes encoding mesenchymal proteins, vimentin mRNA expression increased with severe obstruction at 18 and 36 weeks gestation, while mRNAs for alpha-3, alpha- 4 chains of ColIV and for $\alpha$-SMA were markedly increased in the severely obstructed 36-week gestation kidney when compared to the control normal 18 week kidney (Figure 7(b)).

\section{Discussion}

Epithelial-mesenchymal transition (EMT) and the reciprocal mesenchymal-epithelial transition (MET) are central mechanisms in the development of specialized cells, tissues and organs from a small variety of pluripotent cells in the embryo $[2,16]$. Experimental evidence that has accumulated over the last decade supports the role of EMT in the pathogenesis of many diseases including cancer progression and metastasis [17], and tissue fibrosis associated with interstitial lung [18, 19], liver [20], and kidney disease [4, $5,7]$. The role of EMT has been demonstrated in animal and human studies of postnatal kidney disease, including acquired obstructive nephropathy [21], but there are few data regarding congenital urinary tract obstruction.

Many experimental models of obstructive nephropathy have been developed [22]. These have provided clues to the pathogenesis and pathology of this disease. However, there are some important differences between the human congenital forms of obstruction and experimental models. In human congenital urinary tract obstruction, the timing of obstruction is often early during in utero nephrogenesis as opposed to experimental models, such as the sheep and monkey models, which are mid to late gestation. The bestdescribed models of ureteric obstruction, the mouse and rat, are, in fact, postnatal models. In addition, the severity of obstruction differs. Congenital obstruction can be variable, from severe and complete to mild and partial obstruction. Most experimental models are more controlled and therefore reproducible. Consequently, the histopathology of the human forms of congenital obstruction can be variable (partly due to uncontrollable variability of the timing and severity of obstruction and partly to the inherent bias of the availability of tissue) but given the early onset of obstruction, it is often more severe than that of the experimental forms of postnatal obstruction, particularly the postnatal rodent models, as reflected in the severity of architectural disruption and reduction in glomerular number. There are, however, many histological features shared between both types of 


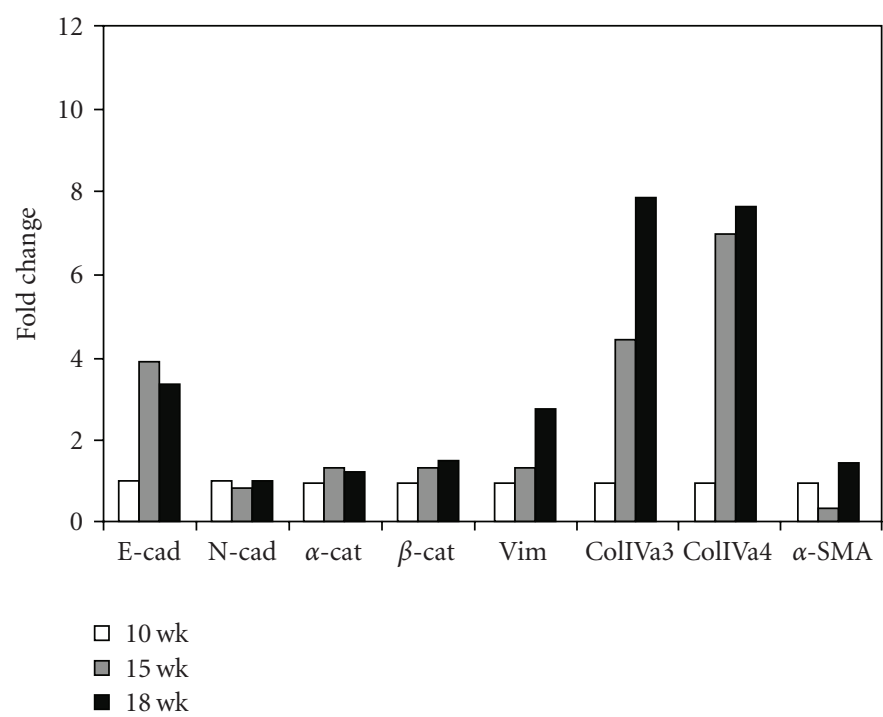

(a)

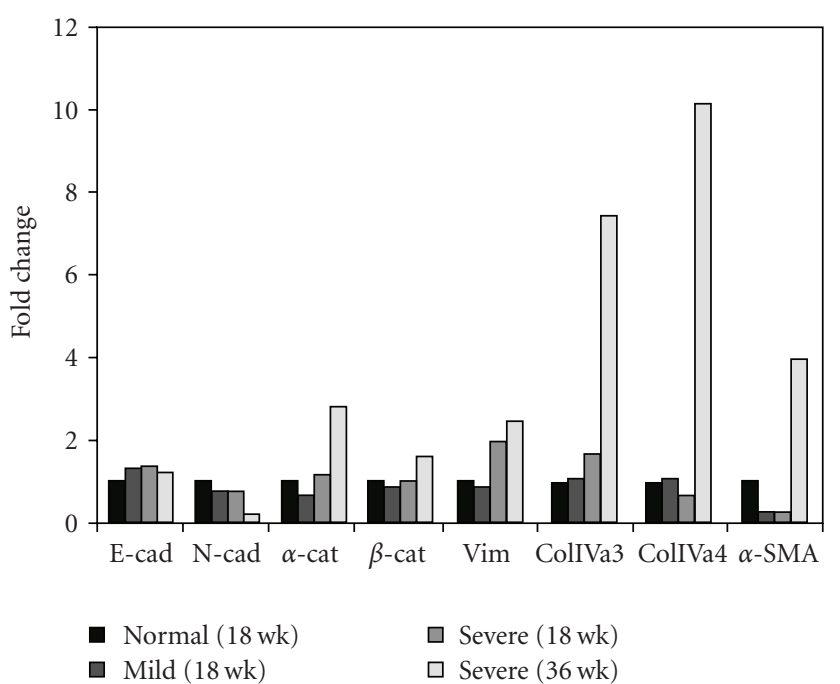

(b)

FIGURE 7: Gene expression in normal and obstructed fetal kidneys. (a) Quantitative PCR analysis of total RNA extracted from 10, 15, and 18 weeks gestation normal kidneys, for genes encoding epithelial-specific (E-cad, N-cad, $\alpha$-cat, and $\beta$-cat) and mesenchymal-specific (vimentin, ColIVa3, ColIVa5 and $\alpha$-SMA) proteins. Values are expressed as fold change in mRNA expression compared to the normal 10 weeks gestation kidney as reference. (b) Quantitative PCR analysis of total RNA extracted from obstructed kidneys at 18 weeks gestation (mild and severe) and 36 weeks gestation (severe), and from 18 weeks normal kidney. Values are expressed as fold change in mRNA expression compared to the normal 18 weeks gestation kidney as reference.

obstruction, most notably human congenital obstruction and in utero models of obstruction such as the sheep and monkey models. These features include extensive reductions in nephron endowment, alterations of tubular and collecting duct development, collecting duct injury, mesenchymal expansion, and interstitial fibrosis.

This report, to our knowledge, is the first description of the phenotypic transition of the obstructed CD epithelium in the human fetus. We have previously described EMT of the obstructed $\mathrm{CD}$ epithelium in a nonhuman primate model of obstructive nephropathy [10]. Similar to the obstructed fetal monkey kidney, the major histological findings in the obstructed human fetal kidney were cystic and dysplastic changes particularly in the medulla, with marked dilatation of the CDs lined by a flattened epithelium. We also demonstrated many of the prerequisite features of CD EMT, including the loss of the cytoskeletal proteins Ecadherin and $\beta$-catenin from cell membranes and intercellular junctions, their translocation into the cytoplasm and nucleus of the obstructed cells, de novo expression of the myoepithelial proteins vimentin and $\alpha$-SMA, and disruption of the obstructed CD epithelial basement membrane. As documented by immunohistochemistry in this study, from early gestation, CD cells express epithelial markers (Ecadherin, $\beta$-catenin) in an intercellular distribution typical of a differentiated epithelium with minimal expression of mesenchymal proteins, such as vimentin and $\alpha$-smooth muscle actin. These findings confirm that the cells have undergone MET. The loss of normal distribution of epithelial markers and de novo expression of mesenchymal proteins by $\mathrm{CD}$ cells in obstructed kidneys imply that obstruction reverses this process. While these findings may not be inclusive of all features of EMT, they do represent a transition to a more mesenchymal phenotype. Our findings would suggest that this transition can occur in cells that have not terminally differentiated. We have also shown that CD dilatation correlated with periductal $\alpha$-SMA expression and with $\mathrm{CD}$ epithelial cell vimentin expression. While we were able to demonstrate a clear correlation in the most severely affected areas, it is important to note that the histopathology and injury in congenital obstructive nephropathy can be variable and focal. We observed areas of severe dysplastic changes alternating with areas of nearly normal histology within the same obstructed kidney. It is therefore very difficult to assign any grading to the whole kidney.

The results of real-time PCR analysis show a marked increase in genes encoding mesenchymal proteins and minimal changes in genes encoding epithelial proteins in obstructed kidneys. It should be noted that we used total RNA extracted from whole kidneys for these experiments. CD epithelial RNA constitutes only a small fraction of the whole kidney RNA. The increase in genes encoding mesenchymal proteins likely reflects mesenchymal expansion and the formation of periductal collars, measures of whole kidney fibrosis, with only a small contribution from the dilated CD epithelial RNA. While the whole kidney fibrosis may be independent of the parallel changes seen in the injured CD epithelium, there is experimental evidence supporting the contribution of the obstructed tubular epithelium to this process [3]. The minimal changes in genes encoding epithelial proteins in the obstructed CDs may be explained again by the relatively small contribution of $\mathrm{CD}$ 

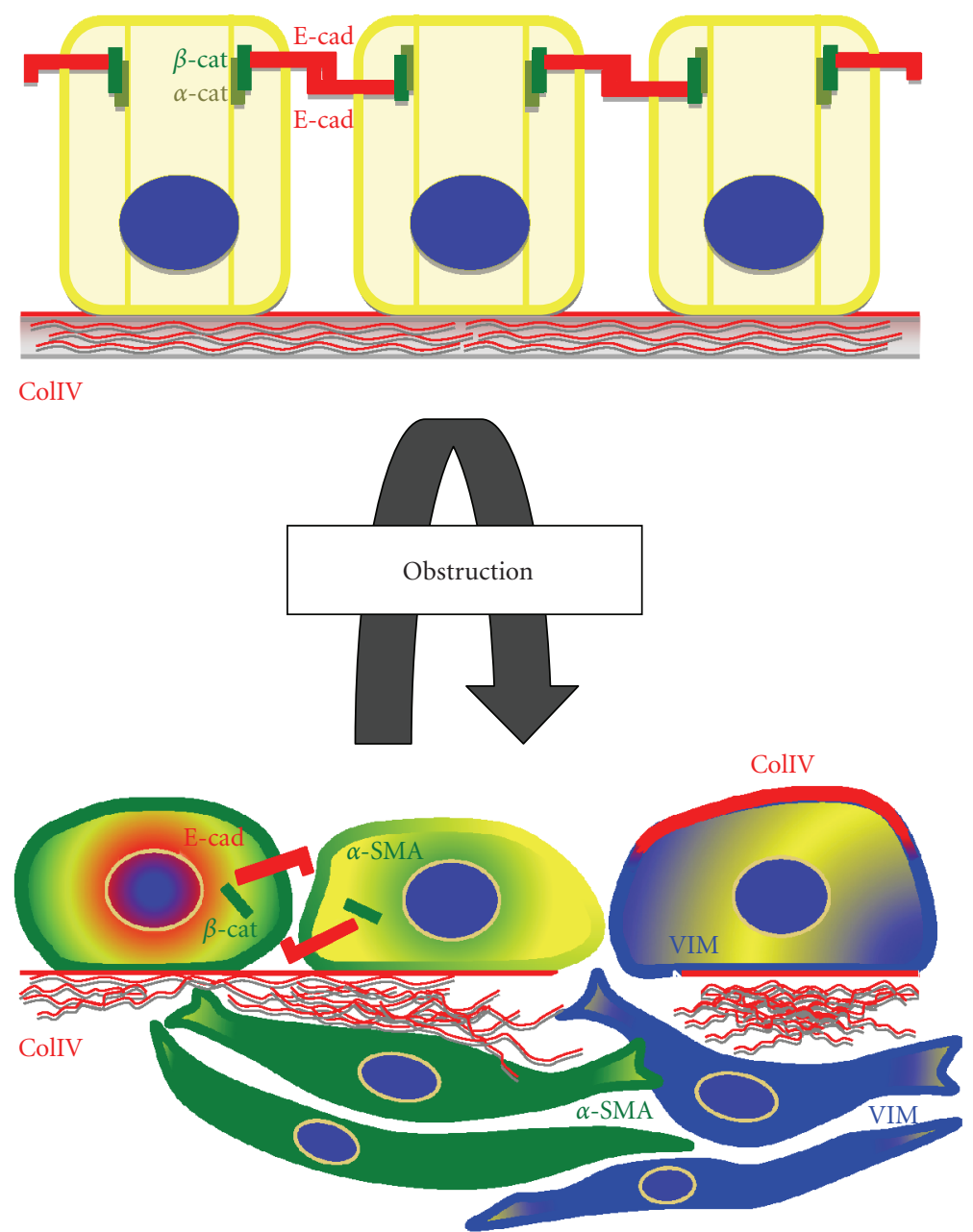

FIGURE 8: Paradigm of changes associated with human congenital urinary tract obstruction. The obstruction of the developing kidneys causes dissociation of the cytoskeletal proteins (E-cadherin, $\beta$-catenin) and their translocation to the cytoplasm and the nucleus. Affected cells become flattened and start expressing mesenchymal markers (vimentin, $\alpha$-SMA) giving them more flexibility and contractility. Breakdown of the ductal basement membrane allows the cells to migrate into the interstitium and to contribute to the myoepthelial population of periductal cells.

RNA relative to total kidney RNA. The other possibility is that there are minimal changes in RNA levels in the obstructed CD epithelium, supported by our immunohistochemistry, demonstrating altered cellular protein localization rather then a decrease in total protein expression.

The sequence of changes seen in EMT is very similar irrespective of the organ involved. The earliest epithelial responses to injury include the loss of intercellular adhesion molecules (E-cadherin, ZO-1) and dissociation of these and other structural anchoring molecules (catenins) from the cytoskeleton of the cell leading to a detachment of the cells from each other, and to their loss of polarity. Reorganization of the actin cytoskeleton, conversion of cytokeratin to vimentin, and de novo expression of $\alpha$-smooth muscle actin, the main features of transition into a myofibroblastic phenotype, provide the transitioning cells with more flexibility and mobility. Vimentin offers the cells more flexibility while maintaining the cell shape and the position of organelles in the cytoplasm [23]. $\alpha$-SMA provides not only structural support but also the element of contractility that can help in the invasiveness of the transformed cells [24]. Breakdown of type IV collagen and laminin in the tubular basement membrane opens the path for these invasive cells to enter the interstitium and become interstitial fibroblasts $[24,25]$. The final nonspecific pathway of this process is the development of tissue fibrosis and destruction of tissue architecture, leading to organ dysfunction and failure (Figure 8). On the other hand, if the disease process is interrupted, phenotypically transformed cells can redifferentiate back into epithelial cells and contribute to the tissue repair, thus undergoing so-called epithelial-mesenchymal-epithelial cycling [26].

\section{Conclusion}

This study describes the phenotypic changes in human congenital obstructive nephropathy. It represents another step in our understanding of the pathogenesis of this 
disease and brings us closer to the identification of the key genes/proteins that play an important role in this condition. Elucidating the role of the individual proteins in EMT will guide the development of targeted treatments to reverse tissue fibrosis and eventually prevent the damage of the affected organ.

\section{Acknowledgments}

This study was supported by award no. P50HL085036 of the National Heart, Lung and Blood Institute of the National Institutes of Health. This work was presented as a poster at the 41st Annual Meeting of the American Society of Nephrology 2008 in Philadelphia.

\section{References}

[1] J. M. Smith, D. M. Stablein, R. Munoz, D. Hebert, and R. A. McDonald, "Contributions of the transplant registry: the 2006 annual report of the north american pediatric renal trials and collaborative studies (NAPRTCS)," Pediatric Transplantation, vol. 11, no. 4, pp. 366-373, 2007.

[2] E. D. Hay and A. Zuk, "Transformations between epithelium and mesenchyme: normal, pathological, and experimentally induced," American Journal of Kidney Diseases, vol. 26, no. 4, pp. 678-690, 1995.

[3] M. Iwano, D. Plieth, T. M. Danoff, C. Xue, H. Okada, and E. G. Neilson, "Evidence that fibroblasts derive from epithelium during tissue fibrosis," The Journal of Clinical Investigation, vol. 110, no. 3, pp. 341-350, 2002.

[4] Y. Liu, "Epithelial to mesenchymal transition in renal fibrogenesis: pathologic significance, molecular mechanism, and therapeutic intervention," Journal of the American Society of Nephrology, vol. 15, no. 1, pp. 1-12, 2004.

[5] M. Zeisberg and R. Kalluri, "The role of epithelial-tomesenchymal transition in renal fibrosis," Journal of Molecular Medicine, vol. 82, no. 3, pp. 175-181, 2004.

[6] N. Picard, O. Baum, A. Vogetseder, B. Kaissling, and M. Le Hir, "Origin of renal myofibroblasts in the model of unilateral ureter obstruction in the rat," Histochemistry and Cell Biology, vol. 130, no. 1, pp. 141-155, 2008.

[7] F. M. Strutz, "EMT and proteinuria as progression factors," Kidney International, vol. 75, no. 5, pp. 475-481, 2009.

[8] A. F. Tarantal, V. K. M. Han, K. C. Cochrum, A. Mok, M. daSilva, and D. G. Matsell, "Fetal rhesus monkey model of obstructive renal dysplasia," Kidney International, vol. 59, no. 2, pp. 446-456, 2001.

[9] D. G. Matsell, A. Mok, and A. F. Tarantal, "Altered primate glomerular development due to in utero urinary tract obstruction," Kidney International, vol. 61, no. 4, pp. 1263-1269, 2002.

[10] M. J. Butt, A. F. Tarantal, D. F. Jimenez, and D. G. Matsell, "Collecting duct epithelial-mesenchymal transition in fetal urinary tract obstruction," Kidney International, vol. 72, no. 8, pp. 936-944, 2007.

[11] E. D. Hay, "The mesenchymal cell, its role in the embryo, and the remarkable signaling mechanisms that create it," Developmental Dynamics, vol. 233, no. 3, pp. 706-720, 2005.

[12] W. C. Prozialeck, P. C. Lamar, and D. M. Appelt, "Differential expression of E-cadherin, N-cadherin and beta-catenin in proximal and distal segments of the rat nephron," $B M C$ Physiology, vol. 4, article 1, pp. 1-36, 2004.
[13] R. Kalluri and E. G. Neilson, "Epithelial-mesenchymal transition and its implications for fibrosis," The Journal of Clinical Investigation, vol. 112, no. 12, pp. 1776-1784, 2003.

[14] R. D. Goldman, S. Khuon, Y. H. Chou, P. Opal, and P. M. Steinert, "The function of intermediate filaments in cell shape and cytoskeletal integrity," Journal of Cell Biology, vol. 134, no. 4, pp. 971-983, 1996.

[15] C. E. Kashtan and A. F. Michael, "Alport syndrome," Kidney International, vol. 50, no. 5, pp. 1445-1463, 1996.

[16] Y. Khew-Goodall and C. Wadham, "A perspective on regulation of cell-cell adhesion and epithelial-mesenchymal transition: known and novel," Cells Tissues Organs, vol. 179, no. 1-2, pp. 81-86, 2005.

[17] M. Guarino, B. Rubino, and G. Ballabio, "The role of epithelial-mesenchymal transition in cancer pathology," Pathology, vol. 39, no. 3, pp. 305-318, 2007.

[18] B. C. Willis, R. M. DuBois, and Z. Borok, "Epithelial origin of myofibroblasts during fibrosis in the lung," Proceedings of the American Thoracic Society, vol. 3, no. 4, pp. 377-382, 2006.

[19] H. Corvol, F. Flamein, R. Epaud, A. Clement, and L. Guillot, "Lung alveolar epithelium and interstitial lung disease," International Journal of Biochemistry and Cell Biology, vol. 41, no. 8-9, pp. 1643-1651, 2009.

[20] O. A. Gressner, M. S. Rizk, E. Kovalenko, R. Weiskirchen, and A. M. Gressner, "Changing the pathogenetic roadmap of liver fibrosis? Where did it start; where will it go?" Journal of Gastroenterology and Hepatology, vol. 23, no. 7, part 1, pp. 1024-1035, 2008.

[21] T. Inoue, H. Okada, T. Takenaka, Y. Watanabe, and H. Suzuki, "A case report suggesting the occurrence of epithelialmesenchymal transition in obstructive nephropathy," Clinical and Experimental Nephrology, vol. 13, no. 4, pp. 385-388, 2009.

[22] D. G. Matsell and A. F. Tarantal, "Experimental models of fetal obstructive nephropathy," Pediatric Nephrology, vol. 17, no. 7, pp. 470-476, 2002.

[23] D. M. Toivola, G.-Z. Tao, A. Habtezion, J. Liao, and M. B. Omary, "Cellular integrity plus: organelle-related and proteintargeting functions of intermediate filaments," Trends in Cell Biology, vol. 15, no. 11, pp. 608-617, 2005.

[24] J. Yang and Y. Liu, "Dissection of key events in tubular epithelial to myofibroblast transition and its implications in renal interstitial fibrosis," American Journal of Pathology, vol. 159, no. 4, pp. 1465-1475, 2001.

[25] H. Acloque, J. P. Thiery, and M. A. Nieto, "The physiology and pathology of the EMT. Meeting on the epithelial-mesenchymal transition," EMBO Reports, vol. 9, no. 4, pp. 322-326, 2008.

[26] S. Ishibe and L. G. Cantley, "Epithelial-mesenchymalepithelial cycling in kidney repair," Current Opinion in Nephrology and Hypertension, vol. 17, no. 4, pp. 379-385, 2008. 

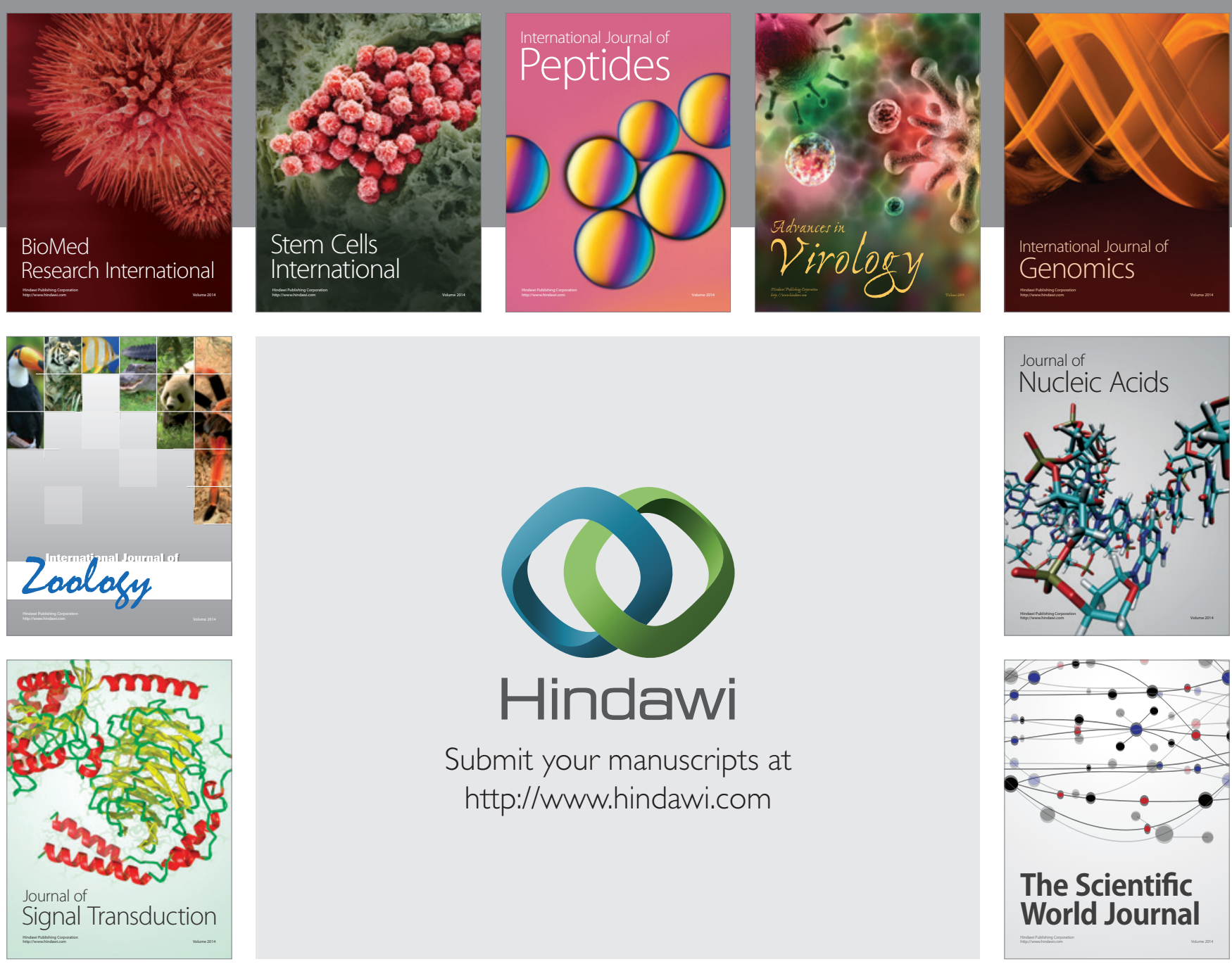

Submit your manuscripts at

http://www.hindawi.com
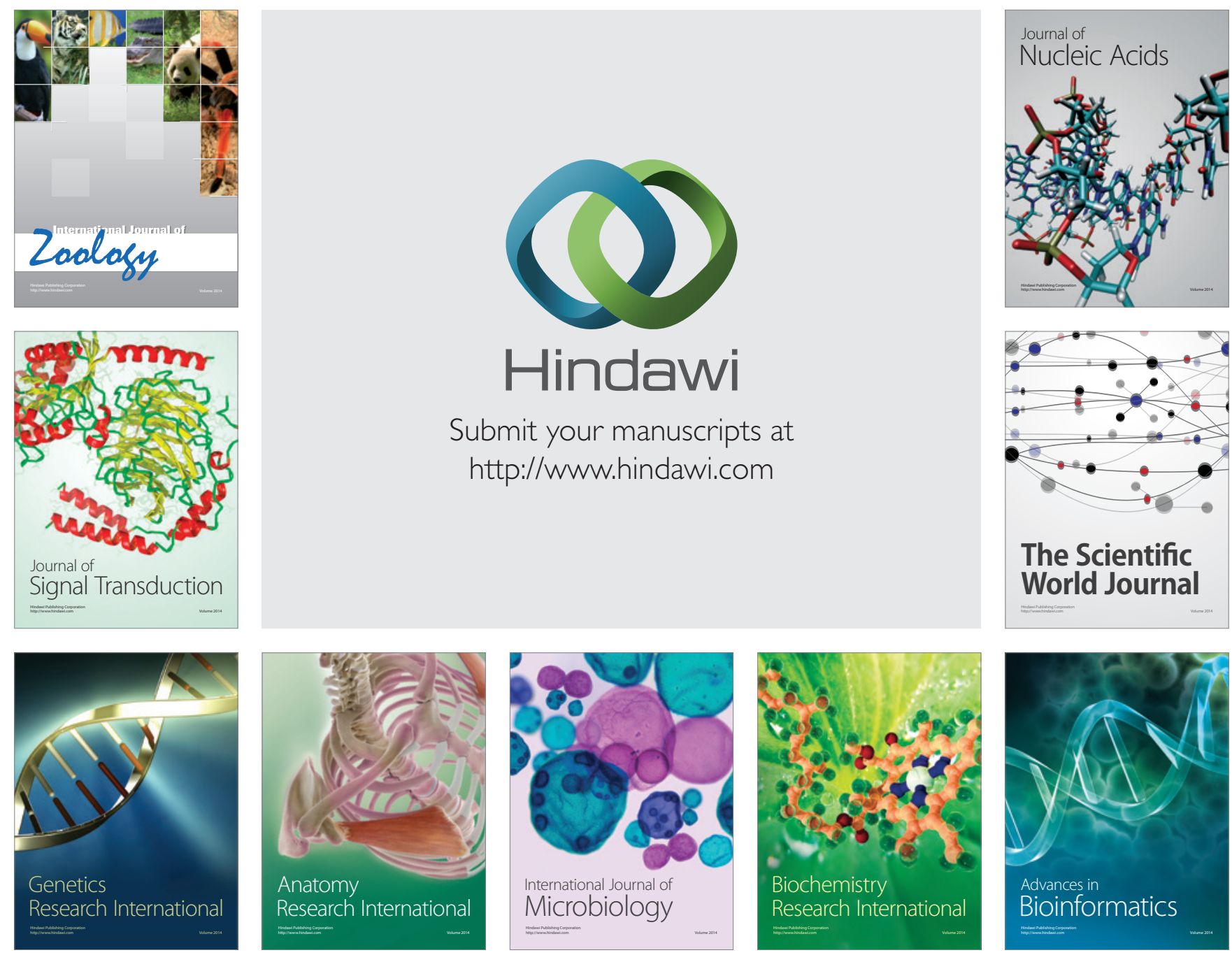

The Scientific World Journal
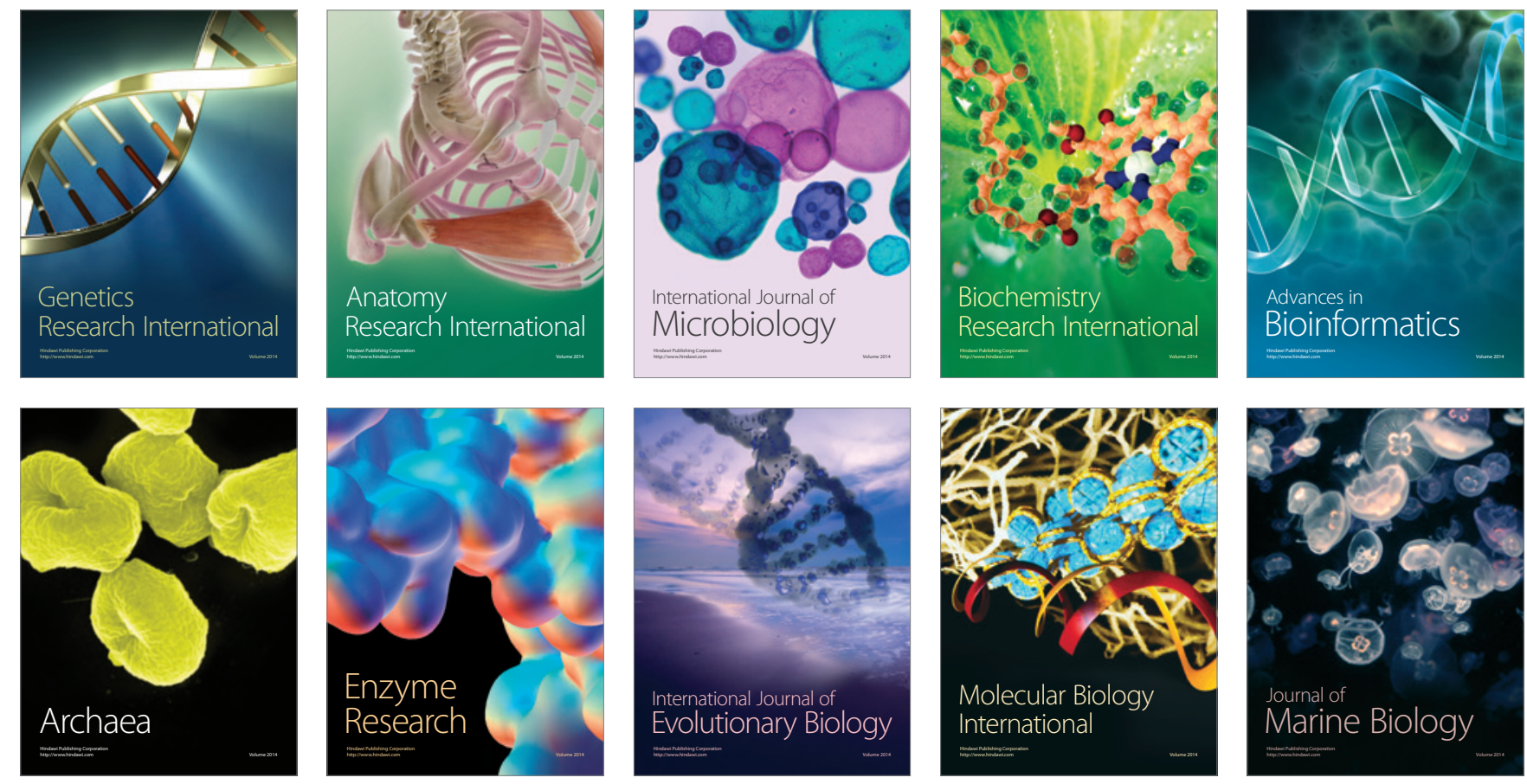